Bifurcation of a reversible Hamiltonian system from a fixed point with fourfold eigenvalue zero

Wagenknecht, Thomas

2002

MIMS EPrint: 2006.380

Manchester Institute for Mathematical Sciences

School of Mathematics

The University of Manchester

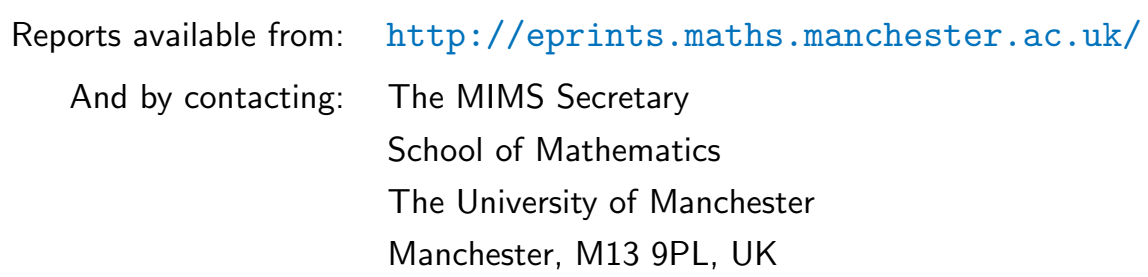

ISSN 1749-9097 


\title{
Bifurcation of a reversible Hamiltonian system from a fixed point with fourfold eigenvalue zero
}

\author{
Thomas Wagenknecht*
}

\begin{abstract}
We study bifurcations from a fixed point with fourfold eigenvalue zero occurring in a two degrees of freedom Hamiltonian system of second order ODEs which is additionally reversible with respect to two different linear involutions. Using techniques from Catastrophe Theory we are led to a codimension 2 problem and obtain two different unfoldings of the singularity related to the hyperbolic and elliptic umbilic, respectively.

The analysis of the unfolded systems is essentially concerned with the existence and properties of homoclinic and heteroclinic orbits. Our studies are motivated by a problem from nonlinear optics concerning the existence of solitons in a $\chi^{2}$-medium.
\end{abstract}

Keywords: codimension two bifurcation, reversible systems, Hamiltonian systems, connecting orbits

AMS classification: $34 \mathrm{Cxx}, 58 \mathrm{Fxx}$

\section{Introduction}

In this paper we consider a class of systems of second order differential equations (ODEs), depending on a parameter $\lambda \in \mathbb{R}^{k}$ close to 0 , that are both reversible and Hamiltonian. We assume that for $\lambda=0$ the origin is an equilibrium and we assume that the linearisation at this equilibrium possesses a fourfold eigenvalue zero. We study what typically happens under (small) perturbations in a neighbourhood of the origin.

In the first part of the paper we derive a suitable description of the situation in terms of an unfolding. The main idea for the procedure yielding both the normal form of the singular system and the respective unfolding is to

*Department of Mathematics, TU Ilmenau, PSF 100565, 98684 Ilmenau, Germany, Email: murmel@mathematik.tu-ilmenau.de, Phone: 00493677 693254, Fax: 00493677693270 
exploit the Hamiltonian structure and to apply Catastrophe Theory to obtain an unfolded normal form of the Hamiltonian. This procedure was successfully applied to planar Hamiltonian systems in $[2,9]$. The outcome in our case are two unfoldings that differ in signs of higher order terms. Using the standard symplectic structure we derive the corresponding two families of ODEs which will be referred to as the (4-dimensional) reversible hyperbolic umbilic and the (4-dimensional) reversible elliptic umbilic, respectively.

The second part of the paper is devoted to an analysis of the unfolded vector fields. We concentrate on the existence of homoclinic and heteroclinic orbits for the systems and study their properties. We start by presenting local bifurcation diagrams showing the fixed points of the systems together with the eigenvalues of their linearisations in dependence on the parameters. Using several techniques from dynamical systems theory we rigorously prove the existence of homoclinic and heteroclinic orbits of the systems. Some important open questions are discussed.

We would like to make clear that we do not intend to give a complete description of the generic dynamics in a neighbourhood of fixed points with fourfold eigenvalue zero. That is to say, we neither look for a universal unfolding of the singularity nor do we want to derive complete parameter-dependent phase portraits of the unfolded systems. Rather, we concentrate on the analysis of some parts of the dynamics of certain 'model systems' for a class of second order ODEs. But nevertheless, it is possible to show that most of our results are indeed generic (compare with the discussion at the end of the paper).

Our studies are motivated by a problem in nonlinear optics dealing with the existence of solitary light waves and kinks in a nonlinear optical medium. In $[5,25]$ it is shown that for a medium with $\chi^{2}$-nonlinearity such waves can be described as homoclinic and heteroclinic orbits of the following system of ODEs:

$$
\begin{aligned}
s \ddot{v} & =\alpha v-\frac{1}{2} w^{2} \\
r \ddot{w} & =w-w v
\end{aligned}
$$

which originates from a system of coupled nonlinear Schrödinger equations. Here, $\alpha$ is a real parameter; $r, s$ are parameters that can only take values \pm 1 . Note that (1) falls into the class of systems studied here. In the case $r=-1$, $s=1$ one is interested in the existence of heteroclinic orbits, and for $\alpha>0$ there is a chance for such a connection as we find two equilibria

$$
\eta^{ \pm}=\left(v_{1}^{ \pm}, \dot{v}_{1}^{ \pm}, w_{1}^{ \pm}, \dot{w}_{1}^{ \pm}\right)=(1,0, \pm \sqrt{2 \alpha}, 0)
$$

on the same level set of the corresponding Hamiltonian.

For $\alpha \geq 8$ the equilibria are of saddle type, i.e. the associated linearisation possesses four real eigenvalues, and one may obtain existence results for 
heteroclinic orbits applying the techniques of Section 4.1.2 of this paper. For $0<\alpha<8$, however, the fixed points are of saddle-focus type and therefore the situation is more involved. Our idea for attacking this problem was to perform a local bifurcation analysis of the degenerate fixed point which gives rise to $\eta^{ \pm}$at $\alpha=0$. A simple calculation shows that the linearisation of this fixed point has a fourfold eigenvalue zero. Unfortunately, it turns out that this special singularity is of infinite codimension (one finds a line of fixed points at $\alpha=0)$. This makes a rigorous analytical treatment rather complicated. So we drop the concrete physical system but, nevertheless, use its properties as an inspiration for the study of a general problem described in the following section.

Finally, it is worth noting that there are only a few studies about degenerate fixed points in $\mathbb{R}^{4}$. We mention work by Iooss [14] who also studies bifurcations from a fixed point with fourfold eigenvalue zero. In the case studied there the eigenvalue has geometric multiplicity one. Being based on normal form theory his approach is more local than the one we take. This is also reflected in the results about connecting orbits.

\section{The general problem}

We are interested in bifurcations from a fixed point occurring in the system of second order ODEs

$$
\begin{array}{ll}
\ddot{v}= & D_{1} V(v, w, \lambda) \\
\ddot{w}=- & D_{2} V(v, w, \lambda)
\end{array}
$$

for the real-valued functions $v(\cdot)$ and $w(\cdot)$. Here $V: \mathbb{R} \times \mathbb{R} \times \mathbb{R}^{k} \rightarrow \mathbb{R}$ is an arbitrary, smooth, real-valued function whose derivative with respect to the $i$ th variable is denoted by $D_{i} V$, and $\lambda$ is the parameter vector whose dimension will be specified later. We restrict our studies to functions $V$ satisfying the following two assumptions:

(V) $V$ is even in the first argument, i.e. it satisfies

$$
V(v, w, \lambda)=V(-v, w, \lambda), \quad \forall(v, w, \lambda) \in \mathbb{R} \times \mathbb{R} \times \mathbb{R}^{k} .
$$

(FP) For $\lambda=0$ the function $V$ vanishes at the origin together with its first and second derivatives.

$$
V(0,0,0)=0, \quad D_{i} V(0,0,0)=0, D_{i j}^{2} V(0,0,0)=0 \quad i, j \in\{1,2\} .
$$

We view (2) as a dynamical system in $\mathbb{R}^{4}$ with phase space variables

$$
x:=\left(x_{1}, x_{2}, x_{3}, x_{4}\right):=(v, \dot{v}, w, \dot{w})
$$


and use the abbreviated form $\dot{x}=f_{V}(x, \lambda)$. Then the special structure of (2) and assumptions (V), (FP) imply the following properties of the vector field.

- For every $\lambda \in \mathbb{R}^{k}$ system (2) is Hamiltonian with

$$
H_{V}(x, \lambda):=H_{V}\left(x_{1}, x_{2}, x_{3}, x_{4}, \lambda\right)=-\frac{1}{2} x_{2}^{2}+\frac{1}{2} x_{4}^{2}+V\left(x_{1}, x_{3}, \lambda\right) .
$$

- For every $\lambda \in \mathbb{R}^{k}$ system (2) is reversible with respect to the linear involution

$$
R_{1}:\left(x_{1}, x_{2}, x_{3}, x_{4}\right) \mapsto\left(x_{1},-x_{2}, x_{3},-x_{4}\right),
$$

that is $R_{1} f_{V}(x, \lambda)=-f_{V}\left(R_{1} x, \lambda\right)$.

- Because of (V) system (2) is reversible with respect to the linear involution

$$
R_{2}:\left(x_{1}, x_{2}, x_{3}, x_{4}\right) \mapsto\left(-x_{1}, x_{2}, x_{3},-x_{4}\right) .
$$

- (FP) completely describes the linearisation of the vector field $f_{V}$ at the origin for $\lambda=0$. We have

$$
\begin{aligned}
f_{V}(0,0) & =0 \\
D_{1} f_{V}(0,0) & =\left(\begin{array}{llll}
0 & 1 & 0 & 0 \\
0 & 0 & 0 & 0 \\
0 & 0 & 0 & 1 \\
0 & 0 & 0 & 0
\end{array}\right) .
\end{aligned}
$$

We will study what typically happens to this singular fixed point under perturbations, i.e. when $\lambda$ is varied. We study local bifurcations from the fixed point, meaning that we are interested in the dynamics of (small) perturbations in a neighbourhood of the origin. However, because of the richness of dynamical phenomena that is expected to arise a complete parameter-dependent phase portrait is beyond our scope. Motivated by the physical problem in the background we concentrate on the existence and properties of connecting orbits in our analysis.

\section{The unfolding procedure}

In order to obtain a normal form and its unfolding for the above problem we perform an unfolding of the corresponding singular Hamiltonian $H_{V}(\cdot, 0)$, applying Catastrophe Theory (see [3] as a general reference concerning this 
subject). This procedure was developed by Broer et.al. [2] who obtained $C^{\infty}$ versal unfoldings of singular fixed points of one degree of freedom Hamiltonian systems in this way (see also [9] for the planar analogue of our problem). In our (4-dimensional) case we cannot prove that the procedure yields versal unfoldings. Nevertheless, there is a geometrical motivation for it.

We are only interested in perturbations of $f_{V}(x, 0)$ that leave the vector field Hamiltonian. Thus, any unfolding of the singular vector field is accompanied by an unfolding of the singular Hamiltonian. Moreover, orbits of Hamiltonian vector fields are part of the level sets of the Hamiltonian. So in order to derive a complete picture of perturbations of the vector field it is necessary to describe the foliation of the level sets of perturbations of the Hamiltonian completely. Translated into the language of Catastrophe Theory this means that for a versal unfolding of a Hamiltonian vector field it is necessary to have a versal unfolding of the Hamiltonian.

For the moment we forget about assumption $(\mathrm{V})$ and look for a $C^{\infty}$-versal unfolding of a suitably chosen normal form of $H_{V}(\cdot, 0)$. Because of the special structure of $H_{V}(\cdot, 0)$ we can apply the Splitting Lemma ([3], p. 125) which is also valid in the context of functions that are invariant with respect to the action of a compact group, see for instance [1]. This shows that in the unfolding process we can concentrate on the unfolding of $V\left(x_{1}, x_{3}, 0\right)$. (In addition we see that the chosen class of systems (2) which leads to the special form of Hamiltonians (3) is appropriate for our problem and imposes no restriction.) Under assumption (FP) the least degenerate singularities in 2 variables are of codimension three, and we have to deal with two different normal forms, namely with the hyperbolic umbilic $V^{-}$and the elliptic umbilic $V^{+}$

$$
V^{-}\left(x_{1}, x_{3}, 0\right)=x_{1}^{2} x_{3}+\frac{1}{3} x_{3}^{3} \quad \text { and } \quad V^{+}\left(x_{1}, x_{3}, 0\right)=x_{1}^{2} x_{3}-\frac{1}{3} x_{3}^{3},
$$

see for instance $[2,3]$. The universal unfoldings of $V^{\mp}$ are given by

$$
V^{\mp}(x, \lambda)=x_{1}^{2} x_{3} \pm \frac{1}{3} x_{3}^{3}+\mu\left(x_{1}^{2} \mp x_{3}^{2}\right)+\nu x_{1}+\kappa x_{3} .
$$

Note that we have labeled the components of the parameter vector $\lambda=$ $(\mu, \kappa, \nu)$. In order to fulfill $(\mathrm{V})$ we set $\nu=0$. Doing so, we are led to a universal $\mathbb{Z}_{2}$-invariant unfolding of $V(\cdot, 0)$, i.e. a universal unfolding within the class of functions that fulfill (V). This has been proved in [9], see also [16]. Finally, after adding the quadratic form we obtain universal unfoldings of the two corresponding normal forms of $H_{V}(\cdot, 0)$. Also introducing the notation $H^{\mp}$ they read

$$
H^{\mp}(x, \lambda)=-\frac{1}{2} x_{2}^{2}+\frac{1}{2} x_{4}^{2}+x_{1}^{2} x_{3} \pm \frac{1}{3} x_{3}^{3}+\mu\left(x_{1}^{2} \mp x_{3}^{2}\right)+\nu x_{1}+\kappa x_{3} .
$$


The outcome of this unfolding procedure is two different systems of ODEs describing perturbations of the singular system. Because of the corresponding Hamiltonian we call them the (4-dimensional) reversible hyperbolic (elliptic) umbilic

$$
\begin{aligned}
& \dot{x}_{1}=x_{2} \\
& \dot{x}_{2}=2 x_{1} x_{3}+2 \mu x_{1} \\
& \dot{x}_{3}=x_{4} \\
& \dot{x}_{4}=-x_{1}^{2} \mp x_{3}^{2} \pm 2 \mu x_{3}-\kappa
\end{aligned}
$$

with Hamiltonian

$$
H^{\mp}(x, \mu, \kappa)=-\frac{1}{2} x_{2}^{2}+\frac{1}{2} x_{4}^{2}+x_{1}^{2} x_{3} \pm \frac{1}{3} x_{3}^{3}+\mu\left(x_{1}^{2} \mp x_{3}^{2}\right)+\kappa x_{3} .
$$

For the corresponding vector fields we will use the abbreviation $f^{\mp}(x, \mu, \kappa)$.

Remark. We emphasise again that although the Hamiltonians $H^{\mp}$ have been unfolded completely it is an open question whether the same holds true for the corresponding vector fields. Nevertheless, we note that the number of parameters in our unfoldings agrees with results by Hoveijn [12] about the linear codimension of the problem.

\section{Analysis of the unfolded systems}

Our analysis of the systems (6) is essentially concerned with the existence of orbits homoclinic and heteroclinic to fixed points. We start by presenting bifurcation diagrams showing the local bifurcations of the fixed points of the systems and the eigenvalues of their linearisations in the $\mu, \kappa$-plane. Afterwards connecting orbits are discussed. A detailed version of this part can be found in [21]. The reader is also referred to [20] as a source for results and terminology concerning reversible ODEs.

We first present the results for the reversible hyperbolic umbilic $f^{-}$in detail; the corresponding results for the elliptic case $f^{+}$, being derived in a similar manner, are listed briefly afterwards.

\subsection{Results for $f^{-}(x, \mu, \kappa)$}

Let us first analyse the fixed points of $f^{-}$and the eigenvalues of their linearisations in dependence on $\mu, \kappa$. The fixed points of $f^{-}$are given by the system of equations

$$
f^{-}(x, \mu, \kappa)=0
$$


This system can be solved directly and we find that there are two fixed points

$$
\xi_{1 / 2}(\mu, \kappa)=\left(0,0, \mu \pm \sqrt{\mu^{2}-\kappa}, 0\right)
$$

if $\kappa \leq \mu^{2}$ and two additional fixed points

$$
\xi_{3 / 4}(\mu, \kappa)=\left(\mp \sqrt{-3 \mu^{2}-\kappa}, 0,-\mu, 0\right)
$$

if $\kappa<-3 \mu^{2}$. The system has no fixed points for $\kappa>\mu^{2}$. (In the sequel we will neglect the dependence of the fixed points on the parameters and only use $\xi_{i}$.)

Most of the information concerning the fixed points of $f^{-}$is compressed in Figure 3 in the appendix. This diagram shows the fixed points of the system in the $\mu, \kappa$-plane and their eigenvalues. (The eigenvalues were obtained using computer algebra programmes.) Since all fixed points are combined in one diagram the presentation is non-standard and we give some explanations.

Each small numbered box in the diagram refers to a region or line in the $\mu, \kappa$-plane as indicated by the arrows. It shows the existing fixed points of the system as well as the position of their eigenvalues for parameter values in this region. We only distinguish the cases "complex", "real", "imaginary" or "zero" eigenvalues. A "•" denotes a single, a " $\times$ " denotes a double eigenvalue (meaning an eigenvalue of algebraic multiplicity 2). Correspondingly, the diagram reflects qualitative relations only.

Local bifurcations of fixed points occur for parameter values on 2 parabolas

$$
\Gamma_{1} \cup \Gamma_{2}:=\left\{\kappa=\mu^{2}\right\} \quad \text { and } \quad \Gamma_{3} \cup \Gamma_{4}:=\left\{\kappa=-3 \mu^{2}\right\} .
$$

For $\kappa=\mu^{2}$ we encounter a Hamiltonian saddle-node bifurcation while for $\kappa=$ $-3 \mu^{2}$ a pitchfork bifurcation takes place. Let us give additional information that cannot be seen in the diagram.

(i) We have that $H^{-}\left(\xi_{3}, \mu, \kappa\right)=H^{-}\left(\xi_{4}, \mu, \kappa\right)=-\frac{4}{3} \mu^{3}-\kappa \mu$ for all $\kappa<-3 \mu^{2}$. There are no other pairs of fixed points lying on the same level set of $H^{-}$. In particular, $\xi_{3}$ and $\xi_{4}$ are the only candidates for a possible connection by a heteroclinic orbit.

(ii) Each fixed point is symmetric with respect to $R_{1}$, i.e. $R_{1} \xi_{i}=\xi_{i}, i=$ $1, \ldots, 4$, while only $\xi_{1}$ and $\xi_{2}$ are also $R_{2}$-symmetric. Moreover, we have $R_{2} \xi_{3}=\xi_{4}$.

We also note that for certain parameter values one finds qualitative changes in the linearisations of fixed points that may lead to a bifurcation of orbits in a neighbourhood of these fixed points. In parameter space we introduce the parabola

$$
\Gamma_{A} \cup \Gamma_{B}:=\left\{\kappa=-4 \mu^{2}\right\}
$$


If the parameters are varied such that this parabola is crossed with decreasing $\kappa$ then no bifurcation of fixed points occurs. But the dynamics in a neighbourhood of $\xi_{3}$ and $\xi_{4}$ changes since the fixed points turn from centres into saddle-foci (if $\mu<0$ ) or from saddles into saddle-foci (if $\mu>0$ ). In the former case $\xi_{3}, \xi_{4}$ lose their stability and a Hamiltonian Hopf Bifurcation occurs.

Finally we remark that a collision of eigenvalues on the real (imaginary) axis can also be found for the fixed point $\xi_{1}\left(\xi_{2}\right)$ if we cross the line $\kappa=0$ for $\mu>0(\mu<0)$. However, here the eigenvalues do not become complex, but pass through each other still remaining on the real (imaginary) axis. In this case the behaviour is generic: With Fix $\left(R_{i}\right):=\left\{x: R_{i} x=x\right\}$ we have $\left\{\xi_{1}, \xi_{2}\right\} \subset \operatorname{Fix}\left(R_{1}\right) \cap \operatorname{Fix}\left(R_{2}\right)$ and so the fixed points cannot have complex eigenvalues.

\subsubsection{Homoclinic orbits for $f^{-}(x, \mu, \kappa)$}

Let us first show the existence of a homoclinic orbit of $f^{-}$with $\kappa<\mu^{2}$. From the symmetries of the system, we derive the following observation.

Lemma 4.1. The linear manifold

$$
P=\left\{x=\left(x_{1}, x_{2}, x_{3}, x_{4}\right) \in \mathbb{R}^{4}: x_{1}=x_{2}=0\right\}
$$

is invariant under the flow of $f^{-}(x, \mu, \kappa)$.

Note that $P$ is just the fixed space of the involution $R_{1} \circ R_{2}$ which is easily seen to be a symmetry of $f^{-}$. Reduced to $P$ we obtain a one degree of freedom Hamiltonian system which can be analysed by discussing the level sets of the corresponding Hamiltonian. In particular for $\kappa<\mu^{2}$, we find a homoclinic orbit $\gamma_{\text {hom }}$ to the fixed point $\xi_{2}$ (see Figure 1 for a geometric impression). This orbit intersects the fixed spaces of both involutions $R_{1}, R_{2}$ and we conclude that $R_{i} \gamma_{\text {hom }}=\gamma_{\text {hom }}$, i.e. the orbit is $R_{i}$-symmetric for $i=1,2$.

Let us discuss $\gamma_{\text {hom }}$ more closely and let us describe the situation within the invariant plane $P$. Restricted to this plane $\xi_{2}$ is a hyperbolic fixed point, and it is fairly easy to see that $\gamma_{h o m}$ is non-degenerate for all $\kappa<\mu^{2}$. (Recall that a homoclinic orbit to a hyperbolic fixed point $\xi$ is called non-degenerate if the tangent spaces of the stable and unstable manifold $W^{s}(\xi), W^{u}(\xi)$ of this fixed point intersect in a one-dimensional space along the orbit.) In particular, this implies that $W^{s}\left(\xi_{2}\right), W^{u}\left(\xi_{2}\right)$ intersect transversally in the corresponding level set of the Hamiltonian in $P$. This argument explains the stable existence of $\gamma_{h o m}$ for $\kappa<\mu^{2}$. We formulate the existence result for $\gamma_{h o m}$ as a Theorem.

Theorem 4.2 ([21]). Consider the reversible hyperbolic umbilic $f^{-}$and suppose that $\kappa<\mu^{2}$. Then there exists an orbit $\gamma_{\text {hom }}$ homoclinic to the fixed point $\xi_{2}$. This orbit is symmetric with respect to $R_{1}$ and $R_{2}$. 
a)

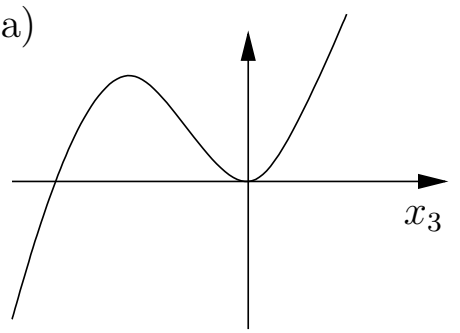

b)

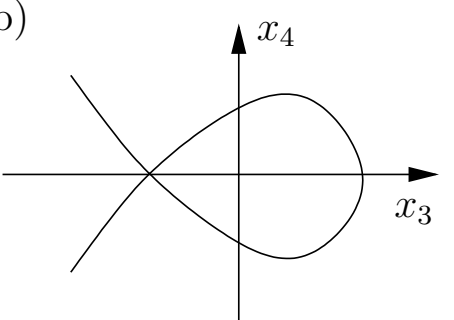

Figure 1: Existence of a homoclinic orbit within $P$ for $\kappa<\mu^{2}$ : Part a) shows qualitatively the potential $V\left(0, x_{3}, \mu, \kappa\right)$ for the Hamiltonian system in $P$, in Part b) $\gamma_{h o m}$ itself is shown.

Let us now analyse the properties of $\gamma_{h o m}$ in the full 4-dimensional phase space. It is clear that the symmetries of the orbit are preserved, since $\gamma_{\text {hom }}$ intersects the fixed spaces of both involutions. Things are more complicated when we want to investigate the non-degeneracy of the orbit. First note that the notion of non-degeneracy is only defined for orbits homoclinic to hyperbolic fixed points. Hence, an investigation of the non-degeneracy of $\gamma_{h o m}$ in $\mathbb{R}^{4}$ only makes sense for parameters between $\Gamma_{2}$ and $\Gamma_{4}$, since this exactly the parameter range where $\xi_{2}$ is hyperbolic. Then we can indeed show that $\gamma_{h o m}$ is nondegenerate in the 4-dimensional phase space. For this we invoke an analytical expression for the homoclinic solution. So let $x_{h o m}(0) \in \operatorname{Fix}\left(R_{1}\right) \cap \operatorname{Fix}\left(R_{2}\right)$ be the (unique) intersection point of $\gamma_{h o m}$ with the fixed spaces of the involutions (again we neglect the dependence of the solution on the parameters). We then have the following expression for the solution (which is valid for all $\kappa<\mu^{2}$ )

$$
x_{\text {hom }}(t)=(0,0, r(t), \dot{r}(t))
$$

with

$$
r(t)=3 \sqrt{\mu^{2}-\kappa} \cdot \operatorname{sech}^{2}\left(\frac{\sqrt[4]{\mu^{2}-\kappa}}{\sqrt{2}} t\right)+\mu-\sqrt{\mu^{2}-\kappa} .
$$

Now we can prove the final result of this part.

Lemma 4.3 ([21]). Let $\gamma_{\text {hom }}$ be as above and consider $f^{-}$with parameter values $\mu>0$ and $-3 \mu^{2}<\kappa<\mu^{2}$. Then $\gamma_{\text {hom }}$ is a non-degenerate homoclinic orbit.

Proof. We will consider the variational equation along $\gamma_{\text {hom }}$ and show that the space of bounded solutions of this equation over $\mathbb{R}$ is one-dimensional. This condition is equivalent to the non-degeneracy of the orbit (see [20]). For $\gamma_{h o m}$ 
the variational equation decouples into two second order ODEs

$$
\begin{array}{ccc}
\ddot{v}= & 2 r(t) v+2 \mu v \\
\ddot{w}= & -2 r(t) w+2 \mu w .
\end{array}
$$

We observe that the second equation is just the variational equation along $\gamma_{\text {hom }}$ within $P$. Since we have non-degeneracy in $P$, the equation therefore possesses a one-dimensional space of bounded solutions.

For the first equation one can show that every bounded solution is squareintegrable. This is a consequence of the exponential dichotomy of (7). Thus, we can multiply this equation through by $v$ and integrate over $\mathbb{R}$ to obtain

$$
-\int_{\infty}^{\infty} \dot{v}^{2} d t=\int_{\infty}^{\infty} 2(r+\mu) v^{2} d t
$$

For every non-trivial bounded solution $v$ the right-hand side is positive, while the left-hand side is negative, which shows that the only bounded solution over $\mathbb{R}$ is the trivial one. This proves the assertion.

We finally note that for parameter values $\kappa<\mu^{2}$ but not between $\Gamma_{2}$ and $\Gamma_{4}$, the fixed point $\xi_{2}$ is non-hyperbolic, namely a saddle-centre. In the generic situation one would expect an orbit homoclinic to a non-hyperbolic fixed point to break up under perturbations. But as we have seen in this section the symmetries of $f^{-}$do not allow this for $\gamma_{\text {hom }}$. Thus, it would be very interesting to analyse the dynamics near the orbit. Such questions, however, will not be pursued further here.

Remark. The results obtained in this section are global in the following sense: The existence of the homoclinic orbit was proved for a large (and determined) range of parameter values. The results are 'maximal' as well. We proved the existence of $\gamma_{h o m}$ for all parameter values where $\xi_{2}$ exists, and the nondegeneracy holds for all parameter values where $\xi_{2}$ is hyperbolic.

\subsubsection{Heteroclinic orbits for $f^{-}(x, \mu, \kappa)$}

Let us now turn to heteroclinic orbits of $f^{-}$. According to observation (i) on page 7 we look for heteroclinic orbits between $\xi_{3}$ and $\xi_{4}$. We note that because of the reversibility of the system with respect to $R_{1}$ and since $\left\{\xi_{3}, \xi_{4}\right\} \subset$ Fix $\left(R_{1}\right)$ such orbits will not come alone but in pairs forming a heteroclinic cycle.

First it is instructive to consider $f^{-}$with parameter values in a neighbourhood of $\Gamma_{4}$. Here $\xi_{2}$ undergoes a pitchfork bifurcation giving rise to 2 saddles 
and turning itself into a saddle centre. In this case centre manifold theory allows to follow the evolution of small bifurcating solutions in a family of reversible planar vector fields and it is well-known that such a reversible pitchfork bifurcation generically gives rise to a heteroclinic cycle (see for instance [18]).

For the actual proof of the existence of a heteroclinic cycle between $\xi_{3}$ and $\xi_{4}$, however, we rather use the fact that $f^{-}$is an indefinite Hamiltonian system (see [11] for the terminology and references). In fact, setting $p=\left(-x_{2}, x_{4}\right)$ and $q=\left(x_{1}, x_{3}\right)$, we can write the Hamiltonian $H^{-}$as

$$
\tilde{H}(q, p)=\frac{1}{2}\langle S p, p\rangle+V(q), \quad(p, q) \in \mathbb{R}^{2} \times \mathbb{R}^{2}
$$

where $\langle S \cdot, \cdot\rangle$ is an indefinite quadratic form. (Note that a Hamiltonian system in $\mathbb{R}^{n} \times \mathbb{R}^{n}$ is called indefinite if the corresponding quadratic form $S$ possesses exactly one negative eigenvalue; but this is automatically satisfied in our case.)

For indefinite Hamiltonian systems there exist powerful results by Hofer and Toland, [11], which ensure the existence of connecting orbits under certain assumptions on the function $V$. For $f^{-}$we will apply Theorem 3 in [11] whose two degrees of freedom version reads as follows.

Theorem 4.4 (Theorem 3, [11]). Consider an indefinite Hamiltonian system

$$
\begin{aligned}
& \dot{q}=\quad S p \\
& \dot{p}=-D V(q)
\end{aligned} \quad(q, p) \in \mathbb{R}^{2} \times \mathbb{R}^{2}
$$

where $S \in G l\left(\mathbb{R}^{2}\right)$ is a self-adjoint linear operator with exactly one negative eigenvalue, and $V: \mathbb{R}^{2} \rightarrow \mathbb{R}$ is smooth. Let $\Sigma$ be the cone defined by

$$
\Sigma:=\left\{q \in \mathbb{R}^{2}:\left\langle S^{-1} q, q\right\rangle<0\right\}
$$

and assume that $V$ satisfies the following assumptions.

(C) There exists a bounded convex set $C \subset \mathbb{R}^{2}$ which is the closure of a component of the set $\left\{q \in \mathbb{R}^{2}: V(q)>0\right\}$ and which has the following properties:

(i) $V(q)>0$ for all $q \in \operatorname{int}(C)$ and there exist exactly two points $a, b \in \partial C$ such that $D V(a)=D V(b)=0$.

(ii) $C \backslash\{a\} \subset a+\Sigma$ and $C \backslash\{b\} \subset b+\Sigma$.

(iii) If $q \in \partial C$ and $D V(q) \neq 0$ and $\langle S D V(q), D V(q)\rangle=0$ then

$$
\left\langle D^{2} V(q) S D V(q), S D V(q)\right\rangle<0 .
$$


Then the system (8) possesses a heteroclinic orbit $\gamma$ which connects the fixed points $(a, 0)$ and $(b, 0)$. Moreover, the $q$-component of $\gamma$ lies in $C$ for all times.

We note that because of the reversibility of (8) with respect to $R:(q, p) \mapsto$ $(q,-p)$ this orbit will also not come alone, but one will in fact find a heteroclinic cycle connecting the fixed points. Now, for $f^{-}$this theorem can be applied to obtain the following result.

Theorem 4.5 ([21]). Consider $f^{-}$with $\mu>0$ and $-4 \mu^{2} \leq \kappa<-3 \mu^{2}$. Then the fixed points $\xi_{3}$ and $\xi_{4}$ are connected by a heteroclinic cycle $\left\{\gamma_{\text {het }}, R_{1} \gamma_{\text {het }}\right\}$ where $\gamma_{\text {het }} \subset W^{u}\left(\xi_{3}\right) \cap W^{s}\left(\xi_{4}\right)$ and $R_{1} \gamma_{h e t} \subset W^{u}\left(\xi_{4}\right) \cap W^{s}\left(\xi_{3}\right)$.

Proof. We will apply Theorem 4.4 to $f^{-}$with fixed parameters $\mu, \kappa$ which requires to find a set $C$ that meets all the requirements in assumption (C) in Theorem 4.4. Using the notation of the theorem we have that

$$
V\left(x_{1}, x_{3}\right)=x_{1}^{2} x_{3}+\frac{1}{3} x_{3}^{3}+\mu\left(x_{1}^{2}-x_{3}^{2}\right)+\kappa x_{3}-\frac{4}{3} \mu^{3}-\kappa \mu
$$

and

$$
\Sigma:=\left\{\left(x_{1}, x_{3}\right) \in \mathbb{R}^{2}:\left|x_{3}\right|<\left|x_{1}\right|\right\} .
$$

Now we take $C$ to be the region bounded by the line $x_{3}=-\mu$ and by the curve $3 x_{1}^{2}+x_{3}^{2}-4 \mu x_{3}+3 \kappa+4 \mu^{2}=0$ (see Figure 2).

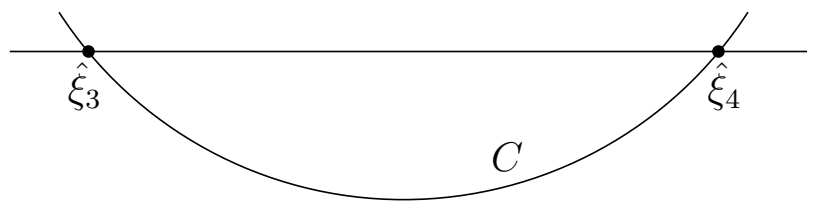

Figure 2: Sketch of the set $C$ required in the proof of Theorem 4.5. By $\hat{\xi}_{3 / 4}$ we denote the projection of $\xi_{3 / 4}$ to $\left(x_{1}, x_{3}\right)$-space.

It is then straightforward to show that $C$ satisfies all of the assumptions in $(\mathrm{C})$ provided that $\mu>0$ and $-4 \mu^{2}<\kappa<-3 \mu^{2}$. Hence, for $f^{-}$there exists a heteroclinic orbit (cycle), say $\gamma_{h e t}$, in this parameter range. But then it is clear that we will also find a heteroclinic orbit (cycle) for $\kappa=-4 \mu^{2}$ which completes the proof.

Generally speaking, it is very hard to perform a rigorous analysis for $\gamma_{\text {het }}$ since we have insufficient information about the orbit. Nevertheless, using estimates based upon the construction of $C$ above we proved in [21] that 
$\gamma_{\text {het }}, R_{1} \gamma_{\text {het }}$ are small bifurcating solutions for parameter values in a neighbourhood below $\Gamma_{4}$. Combining this result with the centre manifold approach sketched at the beginning of this Section yields

Lemma 4.6. There exists a $\delta>0$ such that for $-3 \mu^{2}-\delta<\kappa<-3 \mu^{2}$ the orbits $\gamma_{\text {het }}, R_{1} \gamma_{\text {het }}$ are $R_{2}$-symmetric and form a smooth family parametrised by $\kappa$. There are no other small heteroclinic orbits.

An interesting problem is the existence of heteroclinic orbits of $f^{-}$for parameter values where the fixed points $\xi_{3,4}$ are saddle-foci, i.e. for parameter values below $\Gamma_{A} \cup \Gamma_{B}$. Of course, this is a very hard problem; one possibility for a partial solution is to perform a continuation of $\gamma_{\text {het }}$. This could for instance be done by investigating the non-degeneracy of the orbit which would imply a transverse intersection of the stable and unstable manifolds within the corresponding level set of the Hamiltonian. But still this investigation is too difficult for $\gamma_{h e t}$ since we do not know enough about the orbit. (Recall that for the proof of the non-degeneracy of $\gamma_{h o m}$ in the last section we needed an analytical expression of the homoclinic solution; but now we only know of the existence of a heteroclinic solution.) Fortunately, a continuation of $\gamma_{\text {het }}$ is possible by showing a property of the orbit which is weaker than non-degeneracy. This approach is due to Buffoni [4] who observed that the application of Theorem 4.4 yields orbits which are the result of a topologically transverse intersection of the corresponding stable and unstable manifolds. As the approach requires a rather technical frame we will only present a sketch of the fundamental topological ideas here and refer the reader to [22] for a complete derivation.

The notion of topological transversality is intimately related to the concept of intersection numbers between manifolds. A very good introduction to this can be found in the book by Hirsch, [10], see also [4]. We only note here that an intersection number is a topological object which is stable under small perturbations and under homotopic deformations of the manifolds. Now, two manifolds are said to intersect topologically transversally if the corresponding intersection number is different from zero. By the properties of intersection numbers it can be seen that a topologically transverse intersection cannot be destroyed under small perturbations. However, the notion of topological transversality is weaker than the classic (differential-topological) version of transversality - perturbations of a topologically transverse intersection (that is not transverse in the classic sense) can produce additional intersection points. (Perhaps the best example for this is the (topologically transverse) intersection of the graph of $x \in \mathbb{R} \mapsto x^{3}$ with the $x$-axis.)

Now let us come back to heteroclinic orbits of $f^{-}$. Within the level set of 
$\xi_{3 / 4}$ of the Hamiltonian $H^{-}$we introduce a cross-section

$$
G:=\left\{x=\left(x_{1}, x_{2}, x_{3}, x_{4}\right):\left(x_{1}, x_{3}\right) \in C,\left(x_{2}, x_{4}\right) \in \Sigma, x_{1}=0, x_{2}>0\right\} .
$$

Now it is shown in [22] that the traces of the stable and unstable manifold of $\xi_{4}$ and $\xi_{3}$, respectively, intersect topologically transversally in $G$ provided that $\mu>0$ and $-4 \mu^{2} \leq \kappa<-3 \mu^{2}$. The proof uses assumptions of Theorem 4.4 and the homotopy-invariance of the intersection number. (For technical reasons it also requires to adapt the concept of topological transversality to sets that are not manifolds, but compact pieces of manifolds, see [4,22].) In particular, from the topologically transverse intersection at $\kappa=-4 \mu^{2}$ and from the persistence of such an intersection under small perturbations we obtain

Lemma $4.7([22])$. For all $\mu>0$ there exists $\delta>0$ such that for $-4 \mu^{2}-\delta<$ $\kappa<-4 \mu^{2}$ equation $f^{-}$possesses a heteroclinic cycle connecting $\xi_{3}$ and $\xi_{4}$.

\subsection{Results for $f^{+}(x, \mu, \kappa)$}

We treat the reversible elliptic umbilic in the same way as $f^{-}(x, \mu, \kappa)$ in the last section. Let us start again with the analysis of the fixed points of the system.

If $\kappa>-\mu^{2}$ then there exist two fixed points

$$
\xi_{1 / 2}=\left(0,0, \mu \pm \sqrt{\mu^{2}+\kappa}, 0\right) .
$$

For $\kappa<3 \mu^{2}$ the system has two different fixed points

$$
\xi_{3 / 4}=\left(\mp \sqrt{3 \mu^{2}-\kappa}, 0,-\mu, 0\right) .
$$

We note that $f^{+}$in contrast to $f^{-}$possesses fixed points for each parameter value.

We find the same properties for the fixed points of $f^{+}(x, \mu, \kappa)$ as for those of $f^{-}(x, \mu, \kappa)$. All fixed points are $R_{1}$-symmetric, while only $\xi_{1}$ and $\xi_{2}$ are also symmetric with respect to $R_{2}$. The only fixed points on the same level set of the Hamiltonian $H^{+}$are $\xi_{3}$ and $\xi_{4}=R_{2} \xi_{3}$. As before we have summarised the results about the fixed points of the system in a bifurcation diagram (Figure 4 in the appendix). The following parabolas can be found in this diagram

$$
\Gamma_{4} \cup \Gamma_{3}=\left\{\kappa=3 \mu^{2}\right\}, \Gamma_{1} \cup \Gamma_{2}=\left\{\kappa=-\mu^{2}\right\}, \Gamma_{A} \cup \Gamma_{B}=\left\{\kappa=2 \mu^{2}\right\} .
$$

Also the results about connecting orbits of $f^{+}(x, \mu, \kappa)$ can be obtained in exactly the same way as for $f^{-}(x, \mu, \kappa)$. They read as follows. 
Theorem 4.8. Consider the reversible elliptic umbilic $f^{+}$and suppose $\kappa>$ $-\mu^{2}$. Then there exists an orbit $\gamma_{\text {hom }}$ homoclinic to the fixed point $\xi_{1}$. This orbit is symmetric with respect to $R_{1}$ and $R_{2}$.

The homoclinic solution of $f^{+}$is given by

$$
x_{\text {hom }}(t)=(0,0, r(t), \dot{r}(t))
$$

with

$$
r(t)=-3 \sqrt{\mu^{2}+\kappa} \cdot \operatorname{sech}^{2}\left(\frac{\sqrt[4]{\mu^{2}+\kappa}}{\sqrt{2}} t\right)+\mu+\sqrt{\mu^{2}+\kappa} .
$$

It is also created in a Hamiltonian saddle-node bifurcation. Exactly as for $f^{-}(x, \mu, \kappa)$ we also find a heteroclinic cycle emerging in a reversible pitchfork bifurcation.

Theorem 4.9. Consider $f^{+}$and suppose that $\mu<0$ and $2 \mu^{2} \leq \kappa<3 \mu^{2}$. Then the fixed points $\xi_{3}$ and $\xi_{4}$ are connected by a heteroclinic cycle $\left\{\gamma_{\text {het }}, R_{1} \gamma_{\text {het }}\right\}$ where $\gamma_{\text {het }} \subset W^{u}\left(\xi_{3}\right) \cap W^{s}\left(\xi_{4}\right)$ and $R_{1} \gamma_{\text {het }} \subset W^{u}\left(\xi_{4}\right) \cap W^{s}\left(\xi_{3}\right)$.

\section{Discussion}

In this paper we have studied two systems of ODEs derived as unfoldings of a degenerate fixed point with a fourfold eigenvalue zero. We have presented the diagrams of local bifurcations of fixed points and we have obtained existence results for homoclinic and heteroclinic orbits.

Although no proof has been given that the considered systems are versal unfoldings of the singularity we claim that the presented results are generic. Let us for simplicity restrict the discussion to the hyperbolic case and consider a family of vector fields $g: \mathbb{R}^{4} \times \mathbb{R} \rightarrow \mathbb{R}^{4}$ such that $g(x, 0)=f^{-}(x, 0)$. The fact that the Hamiltonian $H^{-}(x, 0)$ is unfolded completely then immediately shows that there exists a curve $(\mu(\lambda), \kappa(\lambda))$ in parameter space such that $g(x, \lambda)$ and $f(x, \mu(\lambda), \kappa(\lambda))$ have the same number of fixed points of the same type. The results about connecting orbits carry over as well: First we see from $[8,2]$ that within the invariant plane $P$ our procedure yields a versal unfolding of the singular system. In particular, this implies that the results about $\gamma_{\text {hom }}$ are generic. Concerning the heteroclinic cycle we can use the property of topological transversality to infer the existence of heteroclinic cycles also for perturbations of $f^{-}$, see [22].

Let us discuss some open questions concerning the heteroclinic orbits of $f^{-}$. The major problem is the detection of the exact range of parameter values for which heteroclinic orbits between $\xi_{3}$ and $\xi_{4}$ exist. One may try to follow the 
cycle $\left\{\gamma_{\text {het }}, R_{1} \gamma_{\text {het }}\right\}$ under variation of the parameters. Here, recent numerical studies in [17] have shown the existence of a bifurcation curve in parameter space where this cycle becomes degenerate and disappears in a saddle-node bifurcation of heteroclinic cycles. The description of this bifurcation requires techniques different from the ones used in this paper. In addition, the disappearance of the primary cycle does not exclude the possibility that heteroclinic orbits exist for all parameter values $\kappa<-4 \mu^{2}$, i.e. for all parameter values where $\xi_{3,4}$ are saddle-foci. Further existence results might for instance be obtained by variational techniques, as in [15].

It is also interesting to consider the situation around $\Gamma_{A}, \Gamma_{B}$ more closely. On $\Gamma_{A}$ we find a Hamiltonian-Hopf bifurcation of $\xi_{3,4}$, which is well understood locally, regarding the bifurcation of periodic orbits, [19], and small homoclinic orbits (see [13] for results about the corresponding bifurcation in reversible systems). However, assuming the existence of a heteroclinic cycle between $\xi_{3}$ and $\xi_{4}$ for all $\kappa<-4 \mu^{2}$ one has to consider this bifurcation in a global frame and it is not clear what can happen to the cycle.

Of similar interest is the situation for parameter values around $\Gamma_{B}$. Crossing this line gives a situation which - for homoclinic orbits in non-reversible, non-Hamiltonian systems - is sometimes referred to as "broom-bifurcation" (see [7] and references therein). This bifurcation leads to a dramatic change in the dynamics near the cycle from tame to chaotic. In fact, results from [4] show that below $\Gamma_{B}$ the heteroclinic cycle $\left\{\gamma_{\text {het }}, R_{1} \gamma_{\text {het }}\right\}$ is accompanied by infinitely many $n$-homoclinic and $n$-periodic orbits. (Note that this result is very similar to the classic Devaney theorem for non-degenerate homoclinic orbits to saddle-foci in Hamiltonian systems, [24].) It is very interesting to understand how this change of dynamics occurs for the present problem. Some results are available in [23]; see also [6] for an investigation of the related homoclinic case.

Finally, it is worth mentioning that although the systems have been considered as local unfoldings of a degenerate fixed point the results obtained are not chained to the local regime, but can be applied to an arbitrarily large range of parameters by introducing an appropriate scaling, see [17]. Therefore one could use the systems studied here as a starting point for some continuation process to obtain new results for physically relevant problems.

Acknowledgments. Large parts of this paper were contained in the author's diploma thesis. I am indebted to my advisor Jürgen Knobloch for many stimulating discussions. I also would like to thank the referees for their helpful suggestions. 


\section{References}

[1] T. J. Bridges and J. E. Furter 1993. Singularity Theory and Equivariant Symplectic Maps, LNM 1558, Springer.

[2] H. W. Broer, S. N. Chow, Y. Kim, and G. Vegter 1993. A normally elliptic Hamiltonian bifurcation, ZAMP 44, 389-432.

[3] Th. Bröcker and L. Lander 1975. Differentiable Germs and Catastrophes, Cambridge University Press.

[4] B. Buffoni 1999. Shooting methods and topologial tranversality, Proc. Roy. Soc. Edinburgh 129A, 1137-1155.

[5] A. V. Buryak 1996. Solitons due to Quadratic Nonlinearities, PhD thesis, Australian National University.

[6] A. R. Champneys and J. F. Toland 1993. Bifurcation of a plethora of large amplitude homoclinic orbits for autonomous Hamiltonian systems, Nonlinearity 6, 665-721.

[7] A. R. Champneys 1997. Homoclinic orbits in reversible systems and their applications in mechanics, fluids and optics, Physica D 112, 158-186.

[8] M. Golubitsky and I. Stewart 1987. Generic bifurcation of Hamiltonian systems with symmetry, Physica D 24, 391-405.

[9] H. Hanßmann 1997. The reversible umbilic bifurcation, Physica D 112, 81-94.

[10] M. W. Hirsch 1976. Differential Topology, Springer.

[11] H. Hofer and J. F. Toland 1984. Homoclinic, heteroclinic, and periodic orbits for a class of indefinite Hamiltonian systems, Math. Ann. 269, 387403.

[12] I. Hoveijn 1996. Versal deformations and normal forms for reversible and Hamiltonian linear systems, Journal of Differential Equations 126(2), 408-442.

[13] G. Iooss and M.C. Pérouème 1993. Perturbed homoclinic solutions in reversible 1:1 resonance vector fields, Journal of Differential Equations, 102(1), 62-88.

[14] G. Iooss 1995. A codimension 2 bifurcation for reversible vector fields, Fields Institute Communications, Vol. 4, 201-217. 
[15] W. D. Kalies, J. Kwapisz, and R. C. A. M. van der Vorst 1998. Homotopy classes for stable connections between Hamiltonian saddle-focus equilibria. Communications in Mathematical Physics 193, 337-371.

[16] V. Poènaru 1976. Singularités $C^{\infty}$ en présence de symétrie, LNM 510, Springer.

[17] J. Knobloch, T. Rieß, and T. Wagenknecht 2001. A numerical investigation of connecting orbits in a system of second order ODEs, in preparation.

[18] M. A. Teixeira 1997. Singularities of reversible vector fields, Physica $D$ 100, 101-118.

[19] A. Vanderbauwhede 1990. Hopf bifurcation for equivariant conservative and time-reversible systems, Proc. Roy. Soc. Edinburgh 116A, 103-128.

[20] A. Vanderbauwhede and B. Fiedler 1992. Homoclinic period blow-up in reversible and conservative systems, ZAMP 43, 292-318.

[21] T. Wagenknecht 1999. An analytical study of a two degrees of freedom Hamiltonian system associated to the reversible hyperbolic umbilic, Diploma Thesis, TU Ilmenau. Available online at http://imath.mathematik.tu-ilmenau.de/ murmel

[22] T. Wagenknecht 2001. Continuation of heteroclinic cycles via topological transversality, Preprint M 02/01, TU Ilmenau.

[23] T. Wagenknecht 2001. Bifurcation of heteroclinic cycles in reversible systems, in preparation

[24] S. Wiggins 1988. Global Bifurcations and Chaos, Springer.

[25] A. C. Yew 1998. An Analytical Study of Solitary-Waves in Quadratic Media, PhD thesis, Brown University, Providence RI. 


\section{A Bifurcation diagrams of the systems}

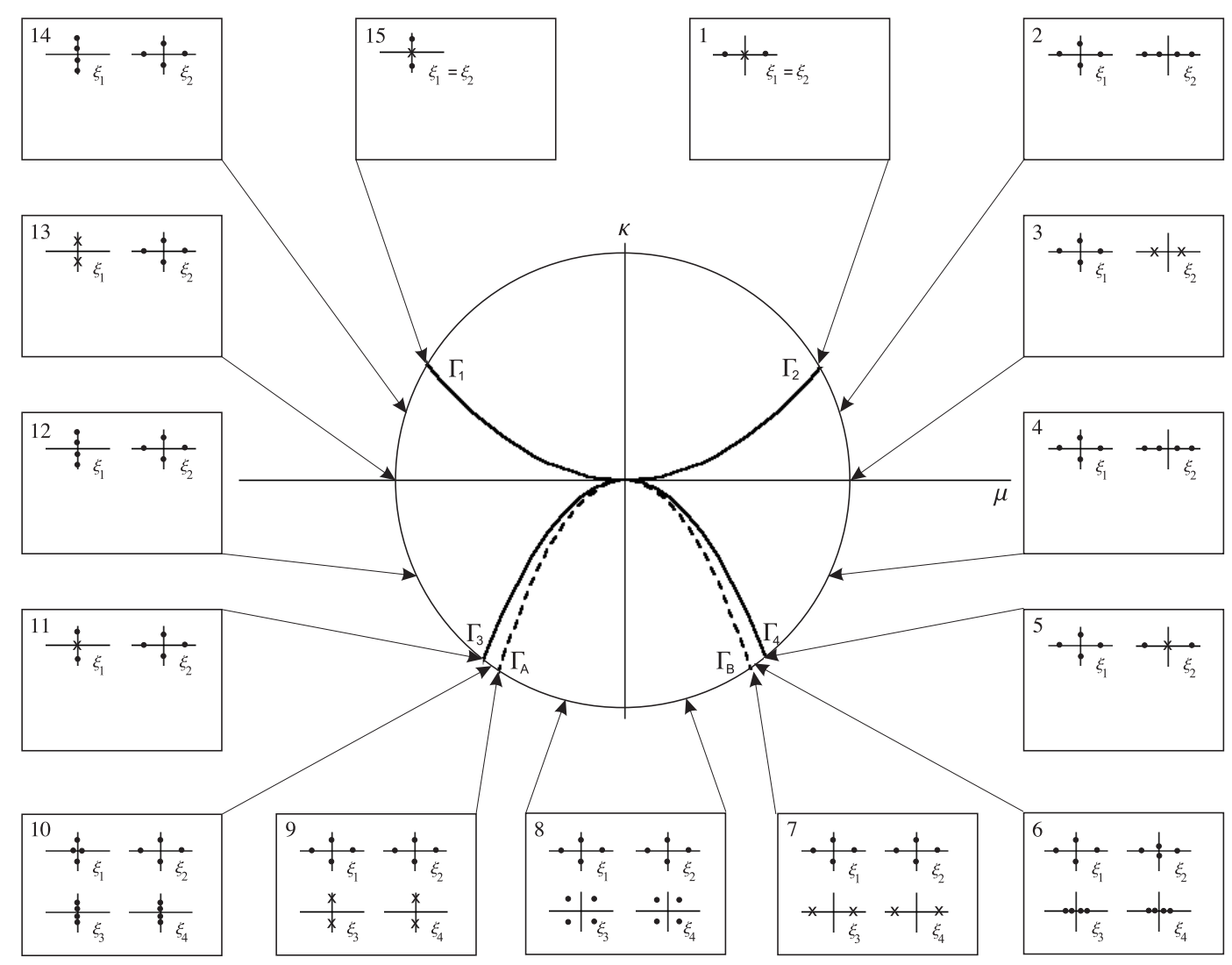

Figure 3: Bifurcation diagram of $f^{-}(x, \mu, \kappa)$ showing the fixed points of the system in the $\mu, \kappa$-plane and the position of their eigenvalues in the complex plane. On the solid curves $\Gamma_{1} \cup \Gamma_{2}:=\left\{\kappa=\mu^{2}\right\}$ and $\Gamma_{3} \cup \Gamma_{4}:=\left\{\kappa=-3 \mu^{2}\right\}$ local bifurcations of fixed points occur, whereas the dotted curve $\Gamma_{A} \cup \Gamma_{B}:=$ $\left\{\kappa=-4 \mu^{2}\right\}$ is related to a qualitative change of the eigenvalues of $\xi_{3 / 4}$. 


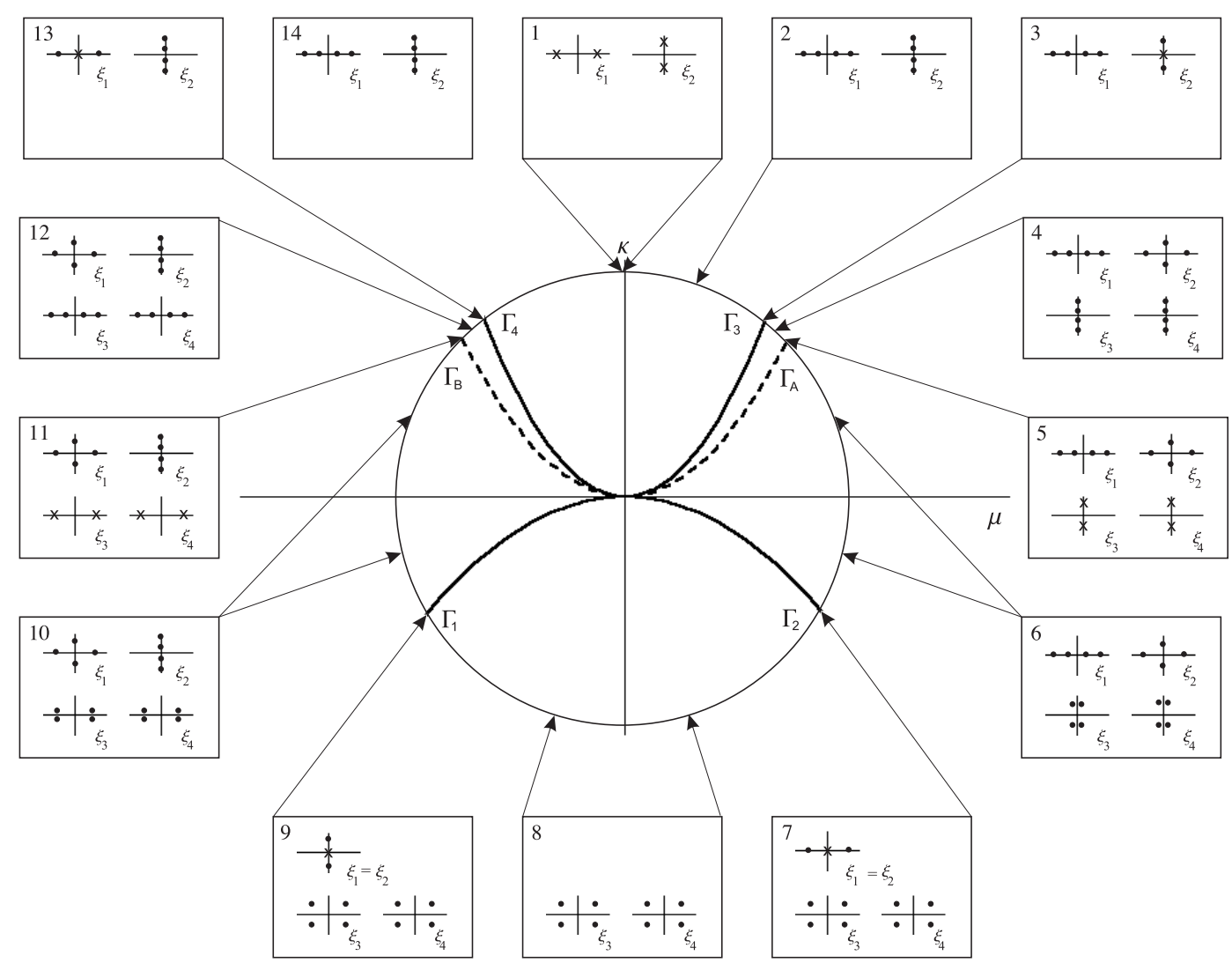

Figure 4: Bifurcation diagram of $f^{+}(x, \mu, \kappa)$ showing the fixed points of the system in the $\mu, \kappa$-plane and the position of their eigenvalues in the complex plane. On the solid curves $\Gamma_{1} \cup \Gamma_{2}:=\left\{\kappa=3 \mu^{2}\right\}$ and $\Gamma_{3} \cup \Gamma_{4}:=\left\{\kappa=-\mu^{2}\right\}$ local bifurcations of fixed points occur, whereas the dotted curve $\Gamma_{A} \cup \Gamma_{B}:=$ $\left\{\kappa=2 \mu^{2}\right\}$ is related to a qualitative change of the eigenvalues of $\xi_{3 / 4}$. 